Актуальность. Интерпретация изменений в легких, выявленных при компьютерной томографии (KT) у пациентов с COVID-19, в некоторых случаях неоднозначна. Сегодня не существует высокодостоверного алгоритма оценки и предсказания течения заболевания на основании рентгенологических данных.

Цель - выявить взаимосвязь рентгенологических симптомов COVID-19 и клинической картины заболевания.

Материал и методы. В обсервационное ретроспективное когортное исследование включены 92 пациента, которые были распределены в 3 группы соответственно клинической тяжести течения COVID-19 (группа легкой COVID-19 29 пациентов, среднетяжелой COVID-19 - 33 и тяжелой COVID-19 - 30). Всем участникам исследования проведена КТ органов грудной клетки при поступлении в стационар и на 10-й день госпитализации.
Результаты. Практически у всех пациентов $(n=28 ; 96,6 \%)$ в группе тяжелой COVID-19 наблюдалось увеличение объема поражения легких с течением заболевания. Риск ухудшения клинической картины у этих пациентов был в 15,037 раза выше в сравнении с пациентами со стабильным объемом поражения легких. На основании данных о распространенности поражения легких можно было с достаточно высокой точностью (площадь под ROC-кривой 0,831, чувствительность 87,5\%, специфичность 70,0\%; $\mathrm{p}<0,001)$ предсказать ухудшение клинического состояния пациентов. Наличие дилатации 6 ронхов в общей группе больных было статистически значимо $(p<0,01)$ связано с увеличением распространенности поражения легких. У 5 (62,5\%) пациентов с выявленной дилатацией бронхов зарегистрировано клиническое ухудшение.

Заключение. Компьютерно-томографический паттерн у пациентов с COVID-19 не всегда коррелирует с клинической тяжестью заболевания. Таким образом, КТ легких не может быть использована для прогнозирования течения COVID-19 без учета данных клинического и лабораторного исследований.

Ключевые слова: COVID-19, новая коронавирусная инфекция, компьютерная томография, вирусная пневмония

Для цитирования: Струтынская АД, Кошурников ДС, Тюрин ИЕ, Карнаушкина МА. Оценка взаимосвязи рентгенологических изменений и степени тяжести заболевания у пациентов с новой коронавирусной инфекцией COVID-19. Альманах клинической медицины. 2021;49(2):171-178. doi: 10.18786/2072-0505-2021-49-028.

Поступила 28.04.2021; доработана 20.05.2021; принята к публикации 21.05.2021; опубликована онлайн 08.06.2021

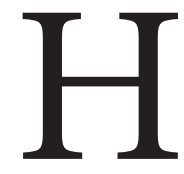
овая коронавирусная инфекция (COVID-19) - заболевание, информация о котором впервые появилась в декабре 2019 г., когда коронавирус SARS$\mathrm{CoV}-2$ был выделен у пациентов в китайском городе Ухань и распространился на территорию государств Европы, Азии, Америки, Африки и Австралии. Подтверждение диагноза основано прежде всего на идентификации вирусной РНК с помощью полимеразной цепной реакции (ПЦР) с обратной транскрипцией. Метод обладает высокой чувствительностью и специфичностью, 
однако не исключает ложноотрицательных результатов [1-6].

Дополнением ПЦР в диагностике COVID-19 служат различные методы лучевой диагностики, прежде всего компьютерная томография (КТ) органов грудной клетки. И хотя установление этиологического диагноза находится вне компетенции методов лучевой диагностики, с их помощью можно определить типичные для COVID-19 изменения в легких или по крайней мере предложить дифференциально-диагностический ряд. КТ характеризуется высокой чувствительностью на ранней стадии заболевания и позволяет при наличии типичных изменений в легких поставить диагноз COVID-19 с высокой вероятностью даже при отрицательных результатах ПЦР [1, 3, 7-9]. Однако у 15-56\% пациентов в первые дни заболевания КТ-картина может быть нормальной, в других случаях при наличии изменений в легких на KT клинические проявления COVID-19 отсутствуют [1, 7, 10-13]. Кроме того, возможно несоответствие клинической тяжести заболевания и объема поражения легких по данным КТ [13-15]. Это создает трудности в интерпретации клинико-рентгенологической картины и говорит о вероятном влиянии индивидуальных факторов на течение COVID-19.

Таким образом, данные литературы свидетельствуют о трудности однозначной интерпретации как объема изменений в легких у пациентов с COVID-19, так и их тяжести и возможной эволюции. Целью нашего исследования было выявить взаимосвязь рентгенологических симптомов COVID-19 и клинической картины заболевания.

\section{Материал и методы}

Обсервационное ретроспективное когортное исследование включало 92 пациентов, выбранных методом простого случайного отбора. Все пациенты добровольно подписали информированное согласие на участие в исследовании. Проведение исследования одобрено на заседании локального этического комитета (протокол № 19-20б от 02.07.2020).

Всем пациентам проводили КТ органов грудной клетки в 1-е сутки поступления в стационар $\left(\mathrm{KT}_{\text {перв }}\right)$ и на 10-е сутки пребывания в стационаре $\left(\mathrm{KT}_{\text {повт }}\right)$ в случае отсутствия показаний для более раннего динамического исследования. Всего проведено 184 КТ-исследования.

На основании Временных методических рекомендаций Министерства здравоохранения Российской Федерации по профилактике,
Струтынская Анастасия Дмитриевна - аспирант кафедры рентгенологии и радиологии хирургического факультета'; ORCID: https://orcid.org/00000001-9325-5587 $\triangle 125993$, г. Москва, ул. Баррикадная, 2/1-1, Российская Федерация. Тел.: +7 (495) 6800599. E-mail: strutynskaya @yandex.ru

Кошурников Дмитрий Сергеевич - канд. мед. наук, заведующий рентгенологическим отделением²; ORCID: https://orcid.org/00000002-7024-9560

\section{Тюрин Игорь}

Евгеньевич - д-р мед. наук, заведующий кафедрой рентгенологии и радиологии хирургического факультета'; ORCID: https://orcid.org/00000003-3931-1431

Карнаушкина Мария Александровна - д-р мед. наук, профессор кафедры внутренних болезней с курсом кардиологии и функциональной диагностики имени академика B.C. Моисеева ${ }^{3}$; ORCID: https://orcid.org/00000002-8791-2920

ФГБОУ ДПО «Российская медицинская академия непрерывного профессионального образования» Минздрава России; 125993, г. Москва,

ул. Баррикадная, 2/1-1, Российская Федерация

2 ГБУЗ г. Москвы

«Госпиталь для ветеранов войн № 3 Департамента здравоохранения города Москвы»; 129336,

г. Москва, ул. Стартовая,

4, Российская

Федерация

${ }^{3}$ ФГАОУ ВО «Российский университет дружбы народов»; 117198,

г. Москва, ул. МиклухоМаклая, 6, Российская Федерация диагностике и лечению новой коронавирусной инфекции (версия 10) пациенты были разделены на 3 группы в зависимости от клинической (без данных КТ) степени тяжести заболевания [16]. Первая группа - легкой COVID-19 - включала 29 пациентов с легким течением заболевания (температура тела ниже $38^{\circ} \mathrm{C}$, кашель, слабость, боли в горле, отсутствие критериев среднетяжелого и тяжелого течения). Во вторую группу среднетяжелой COVID-19 - вошли 33 пациента co среднетяжелым течением COVID-19 (лихорадка выше $38{ }^{\circ} \mathrm{C}$, частота дыхательных движений более 22 в минуту, сатурация кислородом $\left(\mathrm{SpO}_{2}\right)<95 \%$, одышка при физических нагрузках). Третью группу тяжелой COVID-19 составили 30 пациентов с тяжелым и крайне тяжелым течением заболевания (частота дыхательных движений более 30 в минуту, $\mathrm{SpO}_{2} \leq 93 \%$, снижение уровня сознания, ажитация, нестабильная гемодинамика).

КТ выполняли на мультидетекторных сканеpax Toshiba Aquilion One 160 и Toshiba CXL 64 с использованием стандартного протокола: пиковое напряжение на рентгеновской трубке 120 кВ, сила тока 110-250 мАс, толщина среза 2,0, питч 1,33. Параметры легочного и мягкотканого окон: ширина $1500 \mathrm{HU}$, центр -500 HU; ширина $350 \mathrm{HU}$, центр $20 \mathrm{HU}$ соответственно. Сканирование проводили в краниокаудальном направлении от уровня верхушек легких до нижней точки гемидиафрагмы при задержке дыхания на вдохе.

Симптомы по данным КТ дифференцировались согласно определениям сообщества Fleischner [17]:

- «матовое стекло»: повышение плотности легочной ткани с сохранением дифференцировки сосудов и стенок бронхов на ее фоне;

- консолидация: повышение плотности легочной ткани в отсутствие дифференцировки сосудов и стенок бронхов на ее фоне;

- симптом «булыжной мостовой»: утолщение междольковых перегородок на фоне участка «матового стекла»;

- ретикулярный паттерн: утолщение междольковых или внутридольковых перегородок;

- перилобулярный ретикулярный паттерн: утолщение легочного интерстиция вдоль междольковых перегородок.

Для статистической обработки результатов клинико-функционального обследования использовали пакеты программ STATISTICA for Windows v.10.0 (StatSoft Inc., США) и MedCalc 15.2.2 (MedCalc Software). Нормальность распределения проверяли с помощью теста 
Таблица 1. Сравнительная клинико-демографическая характеристика групп пациентов с различной клинической степенью тяжести COVID-19

\begin{tabular}{llll}
\hline Параметр & $\begin{array}{c}\text { Легкая } \\
\text { COVID-19 }(\mathrm{n}=29)\end{array}$ & $\begin{array}{c}\text { Среднетяжелая } \\
\text { COVID-19 }(\mathrm{n}=33)\end{array}$ & $\begin{array}{l}\text { Тяжелая } \\
\text { COVID-19 }(\mathrm{n}=30)\end{array}$ \\
\hline $\begin{array}{l}\text { Пол, } \mathrm{n}(\%) \\
\text { мужчины }\end{array}$ & $15(51,7)$ & $21(63,6)$ & $16(53,3)$ \\
$\begin{array}{l}\text { женщины } \\
\text { Средний возраст, годы, } \mathrm{M} \pm \mathrm{m}\end{array}$ & $44,7 \pm 15,2$ & $12(36,4)$ & $14(46,7)$ \\
$\begin{array}{l}\text { Ухудшение состояния } \\
\begin{array}{l}\text { при динамическом } \\
\text { наблюдении, } \mathrm{n}(\%)\end{array}\end{array}$ & $0^{*}$ & $68,5 \pm 11,7$ & $63,9 \pm 13,2$ \\
\hline
\end{tabular}

"Статистическая значимость различий между группами 1-2 и 1-3

** Статистическая значимость различий между группами 1-2 и 2-3

Колмогорова - Смирнова и Лиллиефорса. Для оценки различия средних в нескольких несвязанных выборках применяли Н-критерий Краскела Уоллиса, сравнение двух независимых групп выполняли с помощью U-критерия Манна - Уитни. Для сравнения категориальных переменных в независимых группах применялся критерий $\chi^{2}$. Для интерпретации дихотомических переменных использованы оценка рисков, отношение шансов и построение ROC-кривой (95\% доверительный интервал). Качественные данные представлены в виде абсолютных или относительных (\%) частот, количественные - в виде средней арифметической и стандартного отклонения $(\mathrm{M} \pm \mathrm{m})$. Результаты считались статистически значимыми при $\mathrm{p}<0,01$.

\section{Результаты}

Демографическая характеристика пациентов дана в табл. 1. В нашем исследовании количество пациентов с хроническими заболеваниями было незначительным во всех группах.

Распространенность поражения легочной паренхимы как при первичном, так и при динамическом исследовании была статистически значимо $(\mathrm{p}<0,001)$ больше у пациентов с тяжелым течением коронавирусной инфекции (рис. 1).

При $\mathrm{KT}_{\text {повт }}$ у 2 (6,9\%) пациентов группы легкой COVID-19 поражение легких было распространенным, с вовлечением в патологический процесс более 50\% легочной паренхимы, при этом клинически они не демонстрировали признаков дыхательной недостаточности (рис. 2). В остальных случаях клинико-рентгенологической диссоциации отмечено не было, поражение легких более $75 \%$ регистрировали только у пациентов группы

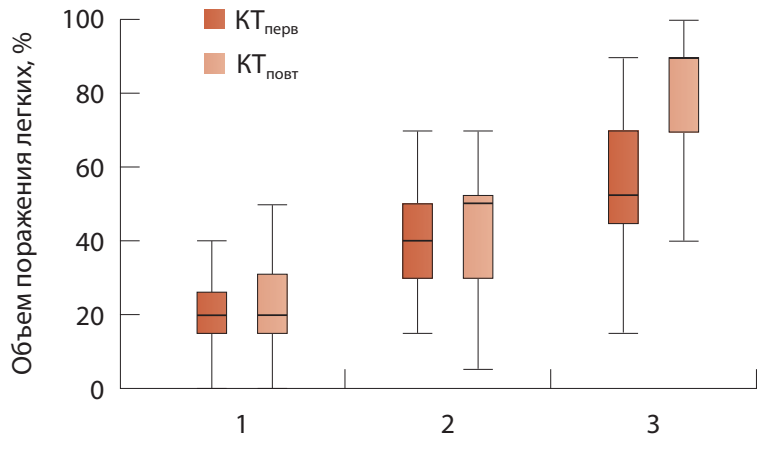

Группы пациентов

Рис. 1. Распространенность поражения легких при первичной $\left(K T_{\text {перв }}\right)$ и динамической (КТ повт компьютерной томографии; 1 - группа легкой COVID-19, 2 - группа среднетяжелой COVID-19, 3 - группа тяжелой COVID-19

тяжелой COVID-19 (n= 14, 46,7\%) при динамической КТ.

Практически у всех пациентов группы тяжелой COVID-19 ( $\mathrm{n}=28,96,6 \%)$ наблюдали нарастание объема изменений в легких на $\mathrm{KT}_{\text {повт }}$. При этом увеличение распространенности поражения в других группах встречалось статистически значимо $(\mathrm{p}<0,001)$ реже: при легкой COVID-19 - у 1 пациента (3,4\%), при среднетяжелой COVID-19 - у 7 пациентов (21,2\%).

B группе легкой COVID-19 не зарегистрировано ни одного случая клинического ухудшения, тогда как у $6(18,2 \%)$ и $26(86,7 \%)$ пациентов групп среднетяжелого и тяжелого течения заболевания соответственно отмечалось нарастание тяжести клинического состояния. Данный параметр имел
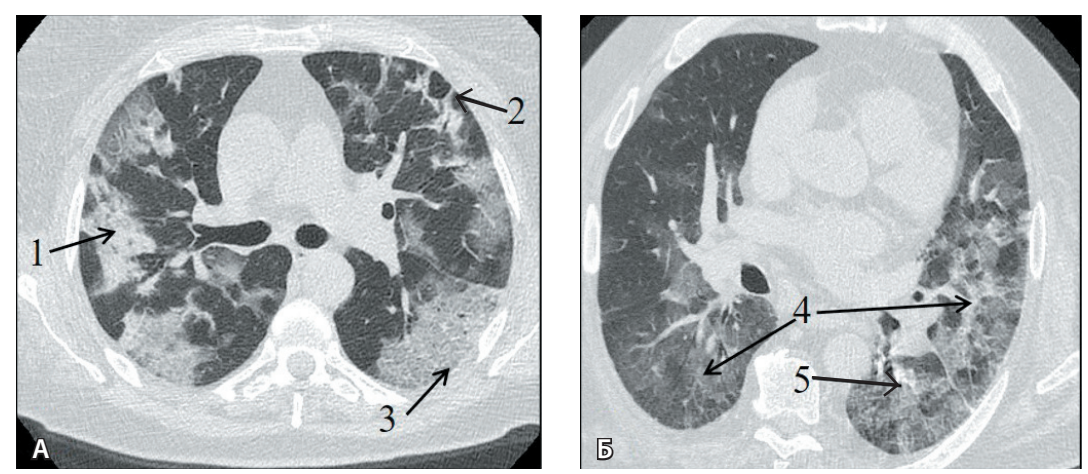

Рис. 2. Пациенты группы легкой COVID-19 с распространенным повреждением легких без признаков дыхательной недостаточности: $\mathbf{A}$ - пациентка Н., 61 год, 15-е сутки заболевания. Отмечается распространенное уплотнение легочной ткани, более выраженное в периферических отделах (площадь поражения около 60\%) (1), с небольшими участками с признаками перилобулярного паттерна (2) и симптома «булыжной мостовой» (3); Б - пациентка Г., 74 года, 15-е сутки заболевания. Отмечаются двусторонние участки «матового стекла» (площадь поражения около 50\%) (4) в сочетании с незначительно утолщенным внутридольковым и междольковым интерстицием в нижней доле левого легкого (5) 
Таблица 2. Сравнительный анализ симптомов, выявленных по данным компьютерной томографии, у пациентов с различной клинической степенью тяжести COVID-19

\begin{tabular}{llll}
\hline Параметр & $\begin{array}{c}\text { Легкая } \\
\text { COVID-19 }(n=29)\end{array}$ & $\begin{array}{c}\text { Среднетяжелая } \\
\text { COVID-19 }(n=33)\end{array}$ & $\begin{array}{l}\text { Тяжелая } \\
\text { COVID-19 }(n=30)\end{array}$ \\
\hline $\mathrm{KT}_{\text {перв }}$ сутки, $\mathrm{M} \pm \mathrm{m}$ & $5,52 \pm 2,76$ & $7,33 \pm 2,70$ & $7,00 \pm 2,70$
\end{tabular}

Результаты первичного КТ-исследования, абс. (\%)

$\begin{array}{llll}\text { нет изменений } & 1(3,4) & 0 & 0 \\ \text { «матовое стекло» } & 19(63,3) & 19(57,6) & 18(62,1) \\ \begin{array}{l}\text { консолидация } \\ \text { сочетание признаков }\end{array} & 7(23,3) & 5(15,2) & 3(10,3) \\ \text { ретикулярный паттерн } & 17(58,6) & 9(27,2) & 8(27,6) \\ \text { «булыжная мостовая» } & 2(6,9) & 20(60,6) & 12(40,0) \\ \text { дилатация бронхов } & 0 * & 8(24,2) & 8(26,7) \\ \text { КТ } & 2(6,1) & 6(20,0) \\ \text { повт сутки, М } \pm \mathrm{m} & 14,9 \pm 4,65 & 17,73 \pm 5,40 & 17,7 \pm 8,39\end{array}$

Результаты динамического КТ-исследования, абс. (\%)

\begin{tabular}{llll} 
«матовое стекло» & $18(60,0)$ & $7(21,2)$ & $10(34,5)$ \\
консолидация & $9(30,0)$ & $19(57,6)$ & $8(27,6)$ \\
$\begin{array}{l}\text { сочетание признаков } \\
\text { «булыжная мостовая» }\end{array}$ & $2(6,7)$ & $7(21,2)$ & $6(20,7)$ \\
$\begin{array}{l}\text { ретикулярный паттерн } \\
\text { дилатация бронхов }\end{array}$ & $13(44,8)$ & 0 & $6(20,7)^{* *}$ \\
$\begin{array}{l}\text { плевральный выпот } \\
\text { организующаяся }\end{array}$ & $0(20,7)^{\dagger}$ & $18(54,5)$ & $21(70,0)$ \\
пневмония & 0 & $2(6,1)$ & $12(40,0)$ \\
\hline
\end{tabular}

КТ - компьютерная томография, КТ 10-е сутки пребывания в стационаре, Организующаяся пневмония - одновременное наличие $\geq 2$ из следующих симптомов: перилобулярный паттерн, линейные участки консолидации; фиброзные тяжи, параллельные плевре; симптом ободка / обратного ободка, Сочетание признаков одновременное наличие консолидации и «матового стекла», сопоставимых по объему

${ }^{*}$ Статистическая значимость различий между группами 1-3

** Статистическая значимость различий между группами 1-3 и 2-3

${ }^{\dagger}$ Статистическая значимость различий между группами 1-2 и 1-3

прямую статистически значимую, хотя и слабую $\left(\chi^{2} 7,165\right.$, коэффициент сопряженности 0,270 , $\mathrm{p}<0,001)$, связь с увеличением объема изменений в легких.

Частота встречаемости КТ-признаков в большинстве случаев межгрупповых различий не имела (табл. 2). В частности, дилатация бронхов

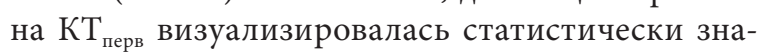
чимо $(\mathrm{p}<0,01)$ чаще среди пациентов группы тяжелой COVID-19, и ее наличие было статистически значимо $(\mathrm{p}<0,01)$ связано с клиническим ухудшением состояния пациентов $(\mathrm{n}=5,62,5 \%)$. У 3 (37,5\%) пациентов данный КТ-признак сочетался с «матовым стеклом», у 1 (12,5\%) - с консолидацией, у 4 (50,0\%) - с сочетанным паттерном. Средний объем изменений в легких у пациентов с дилатацией бронхов на $\mathrm{KT}_{\text {перв }}$ составил $65,6 \pm 12,9 \%$.

При проведении динамической КТ встречаемость КТ-симптомов имела несколько другие значения. В группе тяжелой COVID-19 по сравнению с пациентами других групп статистически значимо $(\mathrm{p}<0,01)$ чаще визуализировался симптом «булыжной мостовой». Дилатацию бронхов выявляли статистически значимо $(\mathrm{p}<0,001)$ чаще среди пациентов группы с тяжелой степенью заболевания по сравнению с пациентами с легкой степенью и у больных группы среднетяжелой COVID-19 по сравнению с легкой. KT-картина организующейся пневмонии в исходе COVID-19 определялась только у пациентов в группах среднетяжелого и тяжелого течения заболевания.

Мы предположили, что КТ-признаки, показавшие статистически значимые межгрупповые различия и/или связь с нарастанием тяжести течения COVID-19 у пациентов, могут быть использованы для предсказания клинического ухудшения состояния (табл. 3). Риск нарастания тяжести клинического состояния оказался в 15 раз выше у пациентов с увеличением объема повреждения легких. При этом если у пациента при поступлении распространенность поражения легочной паренхимы превышала 50\%, риск клинического ухудшения уменьшался практически на 90\%. Подобная закономерность может объясняться изначально тяжелым состоянием этих пациентов. Шансы нарастания тяжести течения заболевания при наличии других КТ-симптомов статистически значимо не изменялись.

Объем поражения легочной паренхимы на $\mathrm{KT}_{\text {перв }}$ показал хорошую предсказательную способность клинического ухудшения с чувствительностью 87,5\% и специфичностью 70,0\% (рис. 3).

\section{Обсуждение}

Проведенное исследование имеет некоторые ограничения. Во-первых, в связи с поступлением большинства пациентов в стационар на 6-7-е сутки от начала заболевания оценка рентгенологической картины на более ранней стадии COVID-19 была невозможна. Во-вторых, оценка объема поражения легочной паренхимы на КТ проводилась 
Таблица 3. Предсказательная способность компьютерно-томографических характеристик для прогнозирования клинического ухудшения

\begin{tabular}{|c|c|c|c|c|c|}
\hline Параметр & Абсолютный риск & $\begin{array}{l}\text { Относительный риск } \\
\text { (95\% ДИ) }\end{array}$ & Значение $p$ & $\begin{array}{l}\text { Отношение шансов } \\
\text { (95\% ДИ) }\end{array}$ & Значение $p$ \\
\hline Нарастание объема поражения & 0,806 & $15,037(4,9431-45,7427)$ & $<0,0001$ & $73,19(17,58-304,707)$ & $<0,0001$ \\
\hline Объем поражения на К $\mathrm{KT}_{\text {перв }}>50 \%$ & 0,492 & $0,1312(0,03352-0,5134)$ & $<0,01$ & $0,00713(0,00156-0,325)$ & $<0,0001$ \\
\hline Консолидация на К $\mathrm{T}_{\text {перв }}$ & 0,364 & $0,6889(0,3522-1,3476)$ & 0,2763 & $0,576(0,2211-1,499)$ & 0,258 \\
\hline Дилатация бронхов на КТ перв & 0,625 & $1,9444(1,0458-3,6154)$ & 0,0356 & $1,2667(0,282-5,693)$ & 0,7579 \\
\hline «Матовое стекло» на КТ перв & 0,625 & $1,0417(0,7424-1,4616)$ & 0,8133 & $1,111(0,4597-2,6858)$ & 0,815 \\
\hline
\end{tabular}

ДИ - доверительный интервал, $\mathrm{KT}_{\text {перв }}$ - компьютерная томография в 1-е сутки поступления в стационар

качественным методом, без использования программного обеспечения для количественной оценки распространенности изменений в легких, что несколько снижало точность измерения. B-третьих, набор пациентов выполнен без учета проводимой терапии. Степень влияния данного фактора оценить затруднительно в связи с отсутствием к настоящему моменту разработанного специфического метода лечения COVID-19.

Наше исследование проводилось без учета лабораторных показателей инфекционного процесса, поскольку КТ, предположительно, представляет собой наиболее чувствительный метод выявления инфекции SARS-CoV-2 на ранней стадии [7-9]. Однако при анализе изображений мы столкнулись с тем, что КТ не отражает в полной мере тяжесть течения COVID-19. Вероятнее всего, причина этой проблемы заключается в том, что всего три компьютерно-томографических
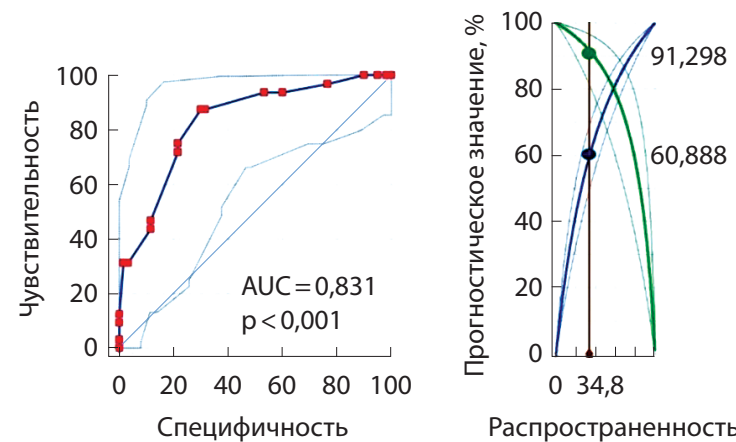

Распространенность болезни, \%

- Положительные прогностические значения

- Отрицательные прогностические значения

Pис. 3. ROC-анализ объема поражения легочной паренхимы на компьютерной томограмме в 1-е сутки исследования; AUC - area under curve (площадь под кривой) симптома - консолидация, «матовое стекло» и ретикулярный паттерн - отражают целый спектр патоморфологических процессов диффузного альвеолярного повреждения (ДАП) [18], в то время как тяжесть ДАП патоморфологически наиболее точно выражается глубиной повреждения альвеолокапиллярного барьера, а не объемом вовлеченной в патологический процесс легочной паренхимы $[18,19]$. Таким образом, становится понятно, что КТ не способна в полной
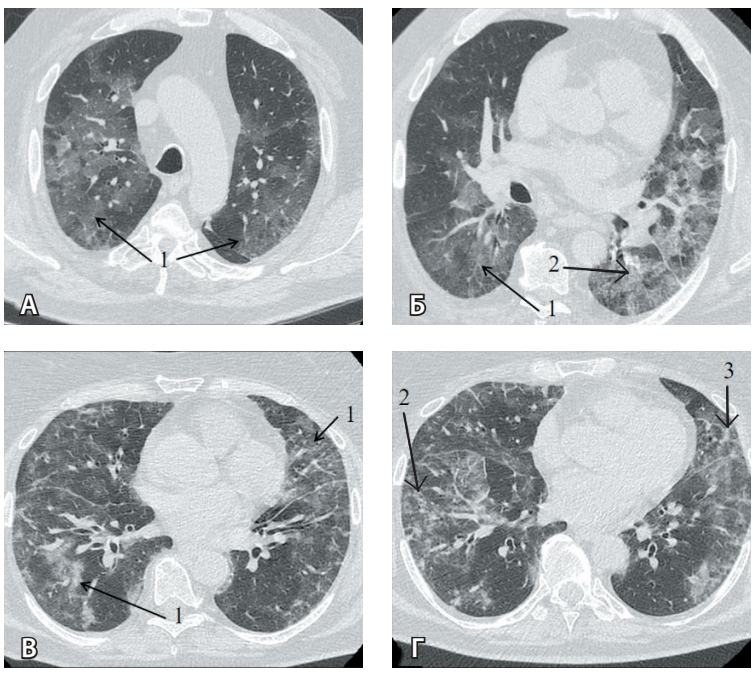

Рис. 4. Демонстрация пациентов с различной клинической картиной на фоне сходных компьютерно-томографических симптомов. Распространенные двусторонние изменения по типу «матового стекла» (1), сочетающиеся с утолщением внутридолькового (2) и междолькового (3) интерстиция: A, Б - пациент K., 71 год, группа тяжелой COVID-19, госпитализирован в отделение реанимации и интенсивной терапии, 14-е сутки заболевания. Дыхательная недостаточность II-III степени. Площадь поражения легочной паренхимы 70\%; В, Г - пациентка Г., 74 года, группа среднетяжелой COVID-19, 15-е сутки заболевания. Дыхательная недостаточность I степени. Площадь поражения легочной паренхимы 70\% 
мере отразить тяжесть течения ДАП и, соответственно, COVID-19. Некоторые клинические наблюдения косвенно подтверждают данный тезис (рис. 4).

Анализ результатов первичной и динамической КТ показал, что частота встречаемости различных КТ-признаков и их распространенность у пациентов с COVID-19 согласуются с данными литературы [10, 12, 14, 20-26].

Мы отметили незначительную разницу во встречаемости организующейся пневмонии при динамической КТ между группами среднетяжелой и тяжелой COVID-19. Частота данного признака была статистически значимо (хоть и незначительно) выше $(\mathrm{p}<0,05)$ среди пациентов со среднетяжелым течением COVID-19. Подобной закономерности в других работах не описано, но можно предположить, что столь слабые статистически значимые различия, вероятнее всего, объясняются не только небольшим объемом выборки, но и более продолжительным течением заболевания у тяжелых пациентов. Именно поэтому к моменту проведения динамической КТ пролиферативные изменения (организующаяся фаза ДАП $[22,23])$ у многих из них не проявились.

Данные литературы относительно прогностической значимости распространенности того или иного КТ-симптома неоднозначны. В работе P. Lyu и соавт. объем консолидации и распространенность симптома «булыжной мостовой» была статистически значимо больше у пациентов с тяжелой COVID-19 по сравнению с легкой формой заболевания [26]. Исследование F. Liu и соавт. показало значимость распространенности «матового стекла» для прогнозирования ухудшения клинического состояния пациентов. При этом наиболее часто встречающимся признаком в данном исследовании был сочетанный паттерн (одновременное наличие «матового стекла» и консолидации в любом соотношении) [27]. Отметим: в этом исследовании первичную и динамическую

\section{Дополнительная информация}

\section{Финансирование}

Работа проведена без привлечения дополнительного финансирования со стороны третьих лиц.

Конфликт интересов

Авторы декларируют отсутствие явных и потенциальных конфликтов интересов, связанных с публикацией настоящей статьи.

\section{Участие авторов}

А.Д. Струтынская - систематизация и статистическая обработка материала, интерпретация результатов исследования, написание текста; Д.С. Кошурников - сбор данных компьютерной томографии,
КТ выполняли в среднем на 4-е и 8-е сутки заболевания соответственно. Тем не менее сравнение результатов приведенных исследований ограничено за счет различных сроков проведения КТ, а также разницы в критериях включения пациентов в исследование.

В ходе проведенного исследования мы получили новые данные о наличии стойкой взаимосвязи дилатации бронхов при КТ тяжести COVID-19, ухудшением клинического состояния и увеличением объема поражения легочной паренхимы. Возможно, наличие дилатации бронхов свидетельствует о более глубоком и распространенном повреждении легочной паренхимы, в том числе легочного интерстиция. По другой версии, дилатация бронхов может свидетельствовать о раннем начале пролиферативной фазы ДАП $[18,19]$. Зафиксированные различия распространенности КТ-симптомов при динамическом исследовании, а также показанная связь дилатации бронхов и симптома «булыжной мостовой» на КТ тяжести заболевания и ухудшением клинического состояния обладают меньшей прогностической ценностью. Однако полученные данные задают вектор дальнейшего исследования клинико-рентгенологической корреляции при COVID-19 и говорят о необходимости прицельного изучения группы пациентов с такими признаками.

\section{Заключение}

Обобщив результаты нашего исследования, можно заключить, что данные КТ не коррелируют в полной мере с клиническими параметрами, что служит следствием самой патоморфологии ДАП. Это свидетельствует о том, что релевантное прогнозирование дальнейшего течения COVID-19 (в частности, на 6-7-е сутки заболевания) может быть сделано только с учетом клинико-лабораторных параметров, значение которых подлежит дальнейшему изучению. (\$) разработка дизайна исследования, редактирование текста; И.Е. Тюрин - разработка дизайна исследования, анализ результатов, редактирование текста, утверждение итогового варианта текста рукописи; М.А. Карнаушкина - концепция и дизайн исследования, сбор клинических данных, интерпретация результатов исследования, редактирование текста, утверждение итогового варианта текста рукописи. Все авторы прочли и одобрили финальную версию статьи перед публикацией, согласны нести ответственность за все аспекты работы и гарантируют, что ими надлежащим образом были рассмотрены и решены вопросы, связанные с точностью и добросовестностью всех частей работы. 


\section{Литература / References}

1.Rubin GD, Ryerson CJ, Haramati LB, Sverzellati N, Kanne JP, Raoof S, Schluger NW, Volpi A, Yim JJ, Martin IBK, Anderson DJ, Kong C, Altes T, Bush A, Desai SR, Goldin O, Goo JM, Humbert M, Inoue $Y$, Kauczor HU, Luo F, Mazzone PJ, Prokop M, Remy-Jardin M, Richeldi L, Schaefer-Prokop CM, Tomiyama N, Wells AU, Leung AN. The Role of Chest Imaging in Patient Management during the COVID-19 Pandemic: A Multinational Consensus Statement from the Fleischner Society. Radiology. 2020;296(1):172-180. doi: 10.1148/radiol.2020201365.

2. Li L, Qin L, Xu Z, Yin Y, Wang X, Kong B, Bai J, Lu Y, Fang Z, Song Q, Cao K, Liu D, Wang G, Xu Q, Fang X, Zhang S, Xia J, Xia J. Using Artificial Intelligence to Detect COVID-19 and Community-acquired Pneumonia Based on Pulmonary CT: Evaluation of the Diagnostic Accuracy. Radiology. 2020;296(2):E65-E71. doi: 10.1148/radiol.2020200905.

3. Xie M, Chen Q. Insight into 2019 novel coronavirus - An updated interim review and lessons from SARS-CoV and MERS-CoV. Int J Infect Dis. 2020;94:119-124. doi: 10.1016/j. ijid.2020.03.071.

4. Ai T, Yang Z, Hou H, Zhan C, Chen C, Lv W, Tao Q, Sun Z, Xia L. Correlation of Chest CT and RT-PCR Testing for Coronavirus Disease 2019 (COVID-19) in China: A Report of 1014 Cases. Radiology. 2020;296(2):E32-E40. doi: 10.1148/radiol.2020200642.

5. Nair A, Rodrigues JCL, Hare S, Edey A, Devaraj A, Jacob J, Johnstone A, McStay R, Denton E, Robinson G. A British Society of Thoracic Imaging statement: considerations in designing local imaging diagnostic algorithms for the COVID-19 pandemic. Clin Radiol. 2020;75(5):329-334. doi: 10.1016/j. crad.2020.03.008.

6. Xie X, Zhong Z, Zhao W, Zheng C, Wang F, Liu J. Chest CT for Typical Coronavirus Disease 2019 (COVID-19) Pneumonia: Relationship to Negative RT-PCR Testing. Radiology. 2020;296(2):E41-E45. doi: 10.1148/radiol.2020200343.

7. Revel MP, Parkar AP, Prosch H, Silva M, Sverzellati N, Gleeson F, Brady A; European Society of Radiology (ESR) and the European Society of Thoracic Imaging (ESTI). COVID-19 patients and the radiology department - advice from the European Society of Radiology (ESR) and the European Society of Thoracic Imaging (ESTI). Eur Radiol. 2020;30(9):4903-4909. doi: 10.1007/s00330-020-06865-y.

8. Simpson S, Kay FU, Abbara S, Bhalla $S$, Chung JH, Chung M, Henry TS, Kanne JP, Kligerman S, Ko JP, Litt H. Radiological Society of North America Expert Consensus Docu- ment on Reporting Chest CT Findings Related to COVID-19: Endorsed by the Society of Thoracic Radiology, the American College of Radiology, and RSNA. Radiol Cardiothorac Imaging. 2020;2(2):e200152. doi: 10.1148/ ryct.2020200152.

9. Sánchez-Oro R, Torres Nuez J, MartínezSanz G. Radiological findings for diagnosis of SARS-CoV-2 pneumonia (COVID-19). Med Clin (Barc). 2020;155(1):36-40. English, Spanish. doi: 10.1016/j.medcli.2020.03.004.

10. Li K, Fang Y, Li W, Pan C, Qin P, Zhong Y, Liu X, Huang M, Liao Y, Li S. CT image visual quantitative evaluation and clinical classification of coronavirus disease (COVID-19). Eur Radiol. 2020;30(8):4407-4416. doi: 10.1007/s00330020-06817-6.

11. Hosseiny M, Kooraki S, Gholamrezanezhad A, Reddy S, Myers L. Radiology Perspective of Coronavirus Disease 2019 (COVID-19): Lessons From Severe Acute Respiratory Syndrome and Middle East Respiratory Syndrome. AJR Am J Roentgenol. 2020;214(5): 1078-1082. doi: 10.2214/AJR.20.22969.

12. Bernheim $A$, Mei $X$, Huang $M$, Yang $Y$, Fayad ZA, Zhang N, Diao K, Lin B, Zhu X, Li K, Li S, Shan H, Jacobi A, Chung M. Chest CT Findings in Coronavirus Disease-19 (COVID-19): Relationship to Duration of Infection. Radiology. 2020;295(3):200463. doi: 10.1148/radiol.2020200463.

13. Chen $\mathrm{H}, \mathrm{Ai}$ L, Lu H, Li H. Clinical and imaging features of COVID-19. Radiol Infect Dis. 2020;7(2):43-50. doi: 10.1016/j. jrid.2020.04.003.

14. Zhang B, Zhang J, Chen H, Yang K, Zhang S. Unmatched clinical presentation and chest CT manifestation in a patient with severe coronavirus disease 2019 (COVID-19). Quant Imaging Med Surg. 2020;10(4):871-873. doi: 10.21037/qims.2020.03.12.

15. Hu X, Chen J, Jiang X, Tao S, Zhen Z, Zhou C, Wang J. CT imaging of two cases of one family cluster 2019 novel coronavirus (2019$\mathrm{nCoV}$ ) pneumonia: inconsistency between clinical symptoms amelioration and imaging sign progression. Quant Imaging Med Surg. 2020;10(2):508-510. doi: 10.21037/ qims.2020.02.10.

16. Министерство здравоохранения Российской Федерации. Временные методические рекомендации: профилактика, диагностика и лечение новой коронавирусной инфекции (COVID-19) [Интернет]. Версия 10 (08.02.2021). Доступно на: https://static-0.minzdrav.gov.ru/system/ attachments/attaches/000/054/588/original/\%D0\%92\%D1\%80\%D0\%B5\%D0\%BC \% D $0 \%$ B $5 \%$ D $0 \%$ B D \% D $0 \%$ B -
D\%D1\%8B\%D0\%B5_\%D0\%9C\%D0\%A0 COVID-19_\%28v.10\%29-08.02.2021 _\%281\%29.pdf. [Ministry of Health of Russian Federation. Temporary methodological recommendations: prevention, diagnostics and treatment of new coronavirus infection (COVID-19) [Internet]. v. 10 (2021 Feb 8). Russian. Available from: https://static-0.minzdrav.gov.ru/system/ attachments/attaches/000/054/588/original/\%D0\%92\%D 1\%80\%D0\%B5\%D0\%BC \% D $0 \%$ B $5 \%$ D $0 \%$ B D \% D $0 \%$ B D\%D1\%8B\%D0\%B5_\%D0\%9C\%D0\%A0_ COVID-19_\%28v.10\%29-08.02.2021 _\%281\%29.pdf.]

17. Hansell DM, Bankier AA, MacMahon H, McLoud TC, Müller NL, Remy J. Fleischner Society: glossary of terms for thoracic imaging. Radiology. 2008;246(3):697-722. doi: 10.1148/radiol.2462070712.

18. Leslie KO. My approach to interstitial lung disease using clinical, radiological and histopathological patterns. J Clin Pathol. 2009;62(5):387-401. doi: 10.1136/ jcp.2008.059782.

19. Kligerman SJ, Franks TJ, Galvin JR. From the radiologic pathology archives: organization and fibrosis as a response to lung injury in diffuse alveolar damage, organizing pneumonia, and acute fibrinous and organizing pneumonia. Radiographics. 2013;33(7):1951-1975. doi: 10.1148/rg.337130057.

20. Kanne JP, Little BP, Chung JH, Elicker BM, Ketai LH. Essentials for Radiologists on COVID-19: An Update-Radiology Scientific Expert Panel. Radiology. 2020;296(2):E113E114. doi: 10.1148/radiol.2020200527.

21. Pan Y, Guan H, Zhou S, Wang Y, Li Q, Zhu T, $\mathrm{Hu} Q$, Xia L. Initial CT findings and temporal changes in patients with the novel coronavirus pneumonia (2019-nCoV): a study of 63 patients in Wuhan, China. Eur Radiol. 2020;30(6):3306-3309. doi: 10.1007/s00330020-06731-x.

22. Pan F, Ye T, Sun P, Gui S, Liang B, Li L, Zheng D, Wang J, Hesketh RL, Yang L, Zheng C. Time Course of Lung Changes at Chest CT during Recovery from Coronavirus Disease 2019 (COVID-19). Radiology. 2020;295(3): 715-721. doi: 10.1148/radiol.2020200370.

23. Liang T, Liu Z, Wu CC, Jin C, Zhao H, Wang Y, Wang Z, Li F, Zhou J, Cai S, Liang Y, Zhou H, Wang $X$, Ren $Z$, Yang J. Evolution of CT findings in patients with mild COVID-19 pneumonia. Eur Radiol. 2020;30(9):4865-4873. doi: 10.1007/s00330-020-06823-8.

24. Wang J, Xu Z, Wang J, Feng R, An Y, Ao W, Gao Y, Wang X, Xie Z. CT characteristics of patients infected with 2019 novel corona- 
virus: association with clinical type. Clin Radiol. 2020;75(6):408-414. doi: 10.1016/j. crad.2020.04.001.

25. Xu X, Yu C, Qu J, Zhang L, Jiang S, Huang D, Chen B, Zhang Z, Guan W, Ling Z, Jiang R, Hu T, Ding Y, Lin L, Gan Q, Luo L, Tang X, Liu J. Imaging and clinical features of patients with 2019 novel coronavirus SARS-CoV-2. Eur
J Nucl Med Mol Imaging. 2020;47(5):12751280. doi: 10.1007/s00259-020-04735-9.

26. Lyu P, Liu X, Zhang R, Shi L, Gao J. The Performance of Chest CT in Evaluating the Clinical Severity of COVID-19 Pneumonia: Identifying Critical Cases Based on CT Characteristics. Invest Radiol. 2020;55(7):412-421. doi: 10.1097/ RLI.0000000000000689.
27. Liu F, Zhang Q, Huang C, Shi C, Wang L, Shi N, Fang $C$, Shan F, Mei X, Shi J, Song F, Yang Z, Ding Z, Su X, Lu H, Zhu T, Zhang Z, Shi L, Shi Y. $C T$ quantification of pneumonia lesions in early days predicts progression to severe illness in a cohort of COVID-19 patients. Theranostics. 2020;10(12):5613-5622. doi: 10.7150/ thno.45985.

\title{
Evaluation of an association of radiological findings and severity of the disease in patients with the new coronavirus infection (COVID-19)
}

\author{
A.D. Strutynskaya $•$ D.S. Koshurnikov² • I.E. Tyurin' \\ M.A. Karnaushkina ${ }^{3}$
}

Rationale: Interpretation of lung abnormalities identified by computed tomography (CT) in patients with COVID-19 could be controversial in some cases. At present, there is no highly reliable algorithm for assessment and prediction of the disease coursed based on CT findings.

Aim: To identify an association of the radiological findings in COVID-19 and its clinical manifestations. Materials and methods: This observational retrospective cohort study included 92 patients, categorized into three groups according to their clinical severity (mild COVID-19 29 patients, moderate COVID-19 33 patients, and severe COVID-19 30 patients). Chest CT was performed in all patients at admittance to the hospital and at day 10 of their hospital stay.

Results: Almost all patients with severe COVID-19 (28 patients, 96.6\%) demonstrated an increase in the damaged lung parenchyma volume at the second CT. The risk of clinical deterioration in these patients was 15.037-fold higher, compared to that in the patients with a stable volume of lung lesions. The area of pulmonary lesions at the first CT demonstrated its good prognostic ability (ROC area under the curve 0.831 , sensitivity $87.5 \%$, specificity $70.0 \%, p<0.001)$ to predict clinical deterioration. The presence of bronchial dilation in the total patient group significantly $(p<0.01)$ correlated with an increase of the pulmonary lesion area. Clinical deterioration was found in 5 patients $(62.5 \%)$ with bronchial dilatation.

Conclusion: CT patterns in COVID-19 patients do not always correlate with clinical severity of the disease. Therefore, lung CT cannot be used for prediction of the COVID-19 course as a single method without clinical and laboratory assessments.

Key words: COVID-19, novel coronavirus infection, computed tomography, viral pneumonia

For citation: Strutynskaya AD, Koshurnikov DS, Tyurin IE, Karnaushkina MA. Evaluation of an association of radiological findings and severity of the disease in patients with the new coronavirus infection (COVID-19). Almanac of Clinical Medicine. 2021;49(2):171-178. doi: 10.18786/2072-0505-2021-49-028.

Received 28 April 2021; revised 20 May 2021; accepted 21 May 2021; published online 8 June 2021

\section{Conflict of interests}

The authors declare no conflict of interest.

\section{Authors' contributions}

A.D. Strutynskaya, systematization and statistical analysis, interpretation of the study results, text writing; D.S. Koshurnikov, computed tomography data collection, the study concept and design, text editing; I.E. Tyurin, the study concept and design, analysis of the results, text editing, approval of the final version of the manuscript; M.A. Karnaushkina, the study concept and design, clinical data collection, interpretation of the study results, text editing, approval of the final version of the manuscript. All the authors have read and approved the final version of the manuscript before submission, agreed to be accountable for all aspects of the work in ensuring that questions related to the accuracy or integrity of any part of the work are appropriately investigated and resolved.

Anastasia D. Strutynskaya - Postgraduate Student, Chair of Roentgenology and Radiology, Faculty of Surgery'; ORCID: https://orcid.org/0000-0001-93255587

$\triangle$ 2/1-1 Barrikadnaya ul., Moscow, 125993, Russian Federation. Tel.: +7 (495) 6800599.

E-mail: strutynskaya@yandex.ru

Dmitry S. Koshurnikov - MD, PhD, Head of Department of Roentgenology²; ORCID: https:// orcid.org/0000-0002-7024-9560

Igor E. Tyurin - MD, PhD, Head of Chair of Roentgenology and Radiology, Faculty of Surgery'; ORCID: https://orcid.org/0000-0003-3931-1431

Maria A. Karnaushkina - MD, PhD, Professor, Chair of Internal Medicine with a Course of Cardiology and Functional Diagnostics named after Academician V.S. Moiseev33 ORCID: https://orcid.org/0000-00028791-2920

\footnotetext{
Russian Medical Academy of Continuous Professional Education; 2/1-1 Barrikadnaya ul. Moscow, 125993, Russian Federation ${ }^{2}$ Hospital for War Veterans No. 3; 4 Startovaya ul., Moscow, 129336, Russian Federation

${ }^{3}$ Peoples' Friendship University of Russia; 6 Miklukho-Maklaya ul., Moscow, 117198, Russian Federation
} 